\title{
Stability of functional differential systems applied to the model of testosterone regulation
}

\author{
Alexander Domoshnitsky ${ }^{1 *}$, Irina Volinsky ${ }^{1}$, Olga Pinhasov and Marina Bershadsky ${ }^{1}$
}

"Correspondence: adom@ariel.ac.il

'Department of Mathematics, Ariel University, Ariel, Israel

\begin{abstract}
In this paper we propose a method for stability studies of functional differential systems. The idea of our method is to reduce the analysis of an $n$-dimensional system to one for an $(n+m)$-dimensional system, where $m$ is a natural number, to obtain stability and then to come back and make conclusions on the stability of the given $n$-dimensional system. As an example, a model describing testosterone by distributed inputs feedback control is considered. The aim of the regulation is to hold testosterone concentration above an appropriate level. The feedback control with integral term is proposed. We have to increase the testosterone level to the normal one. The control we proposed could destroy the stability of the model. That is why we have to choose the parameters of our distributed control, namely a dosage or intensity of assimilation of a medicine in a human body in such a form that the stability of our system is preserved. Thus the problem of regulation of testosterone level leads us to the stability analysis of the functional differential system describing a connection between the concentrations of hormones $(\mathrm{GnRH}),(\mathrm{LH})$, and testosterone (Te). Constructing the system, we discard the connections which seem nonessential. To estimate the effect of these connections is an important problem. We construct the Cauchy matrix of integro-differential system to estimate this influence.
\end{abstract}

Keywords: Exponential stability; Cauchy matrix; Integro-differential systems; Testosterone regulation

\section{Introduction}

Functional differential equation of the form

$$
X^{\prime}(t)+B(t) X(t)+(K X)(t)=f(t), \quad t \in[0, \infty)
$$

where $B(t)$ is an $n \times n$ matrix with essentially bounded coefficients and $K: C^{n} \rightarrow L_{\infty}^{n}$ is a linear bounded operator acting from the space of continuous functions $C^{n}$ to the space of essentially bounded functions $L_{\infty}^{n}, f \in L_{\infty}^{n}$ (all functions we understand as $x:[0, \infty) \rightarrow$ $\mathbb{R}^{n}$ ), appears as a mathematical model describing processes in medicine, biology, and technology [18].

(c) The Author(s) 2019. This article is distributed under the terms of the Creative Commons Attribution 4.0 International License (http://creativecommons.org/licenses/by/4.0/), which permits unrestricted use, distribution, and reproduction in any medium, provided you give appropriate credit to the original author(s) and the source, provide a link to the Creative Commons license, and indicate if changes were made. 
The operator $K$ can be, for example, of the integral form

$$
(K X)(t)=\int_{0}^{t} k(t, s) X(s) d s
$$

Although the control with distributed input control frequently appears as a challenging problem, only a few papers are devoted to it (see, for example, the works [2, 11-14, $16,17,23])$. Noise in the feedback delay control is the main obstacle appearing in mathematical models because of the fact that it is impossible to base our control on the value of process $X(t)$ at a moment $t_{j}$ only, and we have to use an average value of the process $X(t)=\operatorname{col}\left\{x_{1}(t), \ldots, x_{n}(t)\right\}$ at a corresponding neighborhood of the point $t_{j}$. Increasing the number of points, we come actually to the control of the integral form (1.2).

It was demonstrated in the works $[18,21]$ that integro-differential systems can be used to model endocrine regulation in relation to the delay incurred by transport of hormone from the secretion site to receptors. It should be noted (see, for example, [17]) that signaling the receptors is sensitive to the mean value of the hormone concentration over a certain period of time rather than the instantaneous value. Another way to arrange integral terms is, for example, the time required for describing assimilation of medicine. The integral term with a kernel defining a weight of every value adopts this role. It is pointed out in [23] that models with distributed inputs can appear in population dynamics, in propellant rocket motors, and in network control systems. Sufficient conditions of stability for IDE (1.1) were obtained in many well-known works (see, for example, [7, 8, 14, 24, 25, 29]).

In our model, we have to increase the testosterone level to the normal one. The control we proposed could destroy the stability of the model. We have to be sure that the stability of the system is preserved. Note the positivity-based approach to stability of delay equations developed, for example, in the works $[1,9,10,15,19,20,26,28]$. Our method and positivity-based approach can be used also for analysis of nonlinear systems on the basis of the classical approach of the book [22].

\section{Description of the model}

Simplifying the process of testosterone regulation, we can depict the signal transduction pathway initiated in the brain that leads to the testosterone production in the leading cells. This complex pathway encompasses a number of chemical and biological events and can be divided into three stages. The first stage takes place at the brain level. The hypothalamus produces the gonadotrophin releasing hormone $(\mathrm{GnRH})$ that activates the luteinizing hormone (LH) in the pituitary gland. The second stage is reflected by release of LH into the bloodstream. The blood flow transfers the LH hormone to the Leydig cells. The third, final, stage begins with activation of the cascade of biochemical events in Leydig cells that results in production and subsequent release of testosterone [3, 5, 6, 27]. This pathway, as many others in our body, is cyclic and includes the mechanism of negative feedback control. Thus, when the level of testosterone rises, the hypothalamus receives a signal about a sufficient level of hormone and stops producing the GnRH that subsequently inhibits $\mathrm{LH}$ release and as a results leads to lowering the concentration of testosterone. After lowering the level of testosterone, the brain receives a signal about the renewal of the process. Some 
relevant descriptions of the model can be found, for example, in [4-6].

$$
\begin{aligned}
& \left\{\begin{array}{l}
x_{1}^{\prime}(t)+b_{1} x_{1}(t)=0, \\
x_{2}^{\prime}(t)+b_{2} x_{2}(t)-g_{1} x_{1}(t)=0, \\
x_{3}^{\prime}(t)+b_{3} x_{3}(t)-c_{1} \int_{0}^{t} e^{-\alpha_{1}(t-s)} x_{2}(s) d s=0,
\end{array}\right. \\
& x_{1}\left(t_{n}\right)=x_{1}\left(t_{n}^{-}\right)+\lambda_{n}, \quad t_{0}=0, \quad t_{n+1}=t_{n}+\Delta t_{n}, \\
& \Delta t_{n}=\Phi\left(x_{3}\left(t_{n}^{-}\right)\right), \quad \lambda_{n}=F\left(x_{3}\left(t_{n}^{-}\right)\right),
\end{aligned}
$$

where $\Phi$ and $F$ are nondecreasing and nonincreasing functions, respectively.

The model describes an interaction of the concentration of hormones $\mathrm{GnRH}, \mathrm{LH}$, and $\mathrm{Te}$ which will be denoted as $x_{1}, x_{2}$, and $x_{3}$, respectively. The values $b_{i}, 1 \leq i \leq 3$, correspond to the respective half-life times of $\mathrm{GnRH}, \mathrm{LH}$, and $\mathrm{Te}$. In the healthy male body all elements involved in the process work in consent.

We would like to propose a mechanism that allows us to hold the testosterone on a normal level $T(t)$, although the normative exchange of information in the biological system described by the impulses (see the formulas after equation (2.1)) falls and the signals do not enter the brain at all or their influence is not enough to hold the corresponding level of testosterone. We will use the control in the form

$$
u(t)=c_{3} \int_{0}^{t}\left[T(s)-x_{3}(s)\right] e^{-\alpha_{3}(t-s)} d s,
$$

setting it in the right-hand side of the third equation of system (2.1).

The idea is clear: if $T(t)>x_{3}(t)$, the control has to increase the testosterone level $x_{3}(t)$, if $T(t)<x_{3}(t)$, it has to decrease. Thus we come to the third equation in (2.1) of the form

$$
x_{3}^{\prime}(t)+b_{3} x_{3}(t)-c_{1} \int_{0}^{t} e^{-\alpha_{1}(t-s)} x_{2}(s) d s+\left(K x_{3}\right)(t)=f(t)
$$

where the operator $K: C \rightarrow L_{\infty}$ ( $C$ and $L_{\infty}$ are the spaces of continuous and essentially bounded functions respectively) is defined by the equality

$$
\left(K x_{3}\right)(t)=c_{3} \int_{0}^{t} e^{-\alpha_{3}(t-s)} x_{3}(s) d s,
$$

the function $f(t)$ is defined by the equality $f(t)=c_{3} \int_{0}^{t} e^{-\alpha_{3}(t-s)} T(s) d s$, where $T(s)$ is a corresponding "suitable" testosterone concentration.

After substituting control (2.2) in the third equation of system (2.1), we come to the following system:

$$
\left\{\begin{array}{l}
x_{1}^{\prime}(t)+b_{1} x_{1}(t)=0, \\
x_{2}^{\prime}(t)+b_{2} x_{2}(t)-g_{1} x_{1}(t)=0, \\
x_{3}^{\prime}(t)+b_{3} x_{3}(t)-c_{1} \int_{0}^{t} e^{-\alpha_{1}(t-s)} x_{2}(s) d s+c_{3} \int_{0}^{t} e^{-\alpha_{3}(t-s)} x_{3}(s) d s=f(t) .
\end{array}\right.
$$


The corresponding homogeneous system is

$$
\left\{\begin{array}{l}
x_{1}^{\prime}(t)+b_{1} x_{1}(t)=0 \\
x_{2}^{\prime}(t)+b_{2} x_{2}(t)-g_{1} x_{1}(t)=0 \\
x_{3}^{\prime}(t)+b_{3} x_{3}(t)-c_{1} \int_{0}^{t} e^{-\alpha_{1}(t-s)} x_{2}(s) d s+c_{3} \int_{0}^{t} e^{-\alpha_{3}(t-s)} x_{3}(s) d s=0
\end{array}\right.
$$

As usual, the coefficients are supposed to be positive, i.e.,

$$
\alpha_{1}>0, \quad \alpha_{3}>0, \quad b_{1}>0, \quad b_{2}>0, \quad b_{3}>0, \quad c_{3}>0 .
$$

\section{Stability of integro-differential system}

Our approach is based on the fact that system (2.4) of integro-differential equations can be reduced to the following system of ordinary differential equations:

$$
\left\{\begin{array}{l}
x_{1}^{\prime}(t)+b_{1} x_{1}(t)=0 \\
x_{2}^{\prime}(t)+b_{2} x_{2}(t)-g_{1} x_{1}(t)=0 \\
x_{3}^{\prime}(t)+b_{3} x_{3}(t)-c_{1} x_{5}(t)+c_{3} x_{4}(t)=f(t) \\
x_{4}^{\prime}(t)+\alpha_{3} x_{4}(t)-x_{3}(t)=0 \\
x_{5}^{\prime}(t)+\alpha_{1} x_{5}(t)-x_{2}(t)=0
\end{array}\right.
$$

The corresponding homogeneous system is the following:

$$
\left\{\begin{array}{l}
x_{1}^{\prime}(t)+b_{1} x_{1}(t)=0 \\
x_{2}^{\prime}(t)+b_{2} x_{2}(t)-g_{1} x_{1}(t)=0 \\
x_{3}^{\prime}(t)+b_{3} x_{3}(t)-c_{1} x_{5}(t)+c_{3} x_{4}(t)=0 \\
x_{4}^{\prime}(t)+\alpha_{3} x_{4}(t)-x_{3}(t)=0 \\
x_{5}^{\prime}(t)+\alpha_{1} x_{5}(t)-x_{2}(t)=0
\end{array}\right.
$$

Lemma 3.1 The solution-vectors $\operatorname{col}\left(x_{1}(t), x_{2}(t), x_{3}(t)\right)$ of system (2.4) and three first components of the solution-vector $\operatorname{col}\left(x_{1}(t), x_{2}(t), x_{3}(t), x_{4}(t), x_{5}(t)\right)$ of system (3.1) satisfying the initial condition $x_{4}(0)=0, x_{5}(0)=0$ coincide.

If we write system (3.2) in the form $X^{\prime}(t)=A X(t)$, then the matrix of the coefficients is the following:

$$
A=\left(\begin{array}{ccccc}
-b_{1} & 0 & 0 & 0 & 0 \\
g_{1} & -b_{2} & 0 & 0 & 0 \\
0 & 0 & -b_{3} & -c_{3} & c_{1} \\
0 & 0 & 1 & -\alpha_{3} & 0 \\
0 & 1 & 0 & 0 & -\alpha_{1}
\end{array}\right)
$$

Theorem 3.1 System (2.5) is exponentially stable if inequalities (2.6) are true.

Proof The characteristic polynomial of system (3.2) is of the form

$$
P(\lambda)=\left(-b_{1}-\lambda\right)\left(-b_{2}-\lambda\right)\left(-\alpha_{1}-\lambda\right)\left[\lambda^{2}+\left(b_{3}+\alpha_{3}\right) \lambda+\alpha_{3} b_{3}+c_{3}\right] .
$$


It is clear that all the roots

$$
\begin{aligned}
& \lambda_{1}=-b_{1}, \quad \lambda_{2}=-b_{2}, \quad \lambda_{3}=-\alpha_{1}, \\
& \lambda_{4}=\frac{-\left(\alpha_{3}+b_{3}\right)+\sqrt{\left(\alpha_{3}-b_{3}\right)^{2}-4 c_{3}}}{2}, \\
& \lambda_{5}=\frac{-\left(\alpha_{3}+b_{3}\right)-\sqrt{\left(\alpha_{3}-b_{3}\right)^{2}-4 c_{3}}}{2}
\end{aligned}
$$

of $P(x)=0$ have negative real parts. It means, according to Lemma 3.1, the exponential stability of (2.5).

Remark 3.1 We would like to stress that the control in the form (2.2) in the case of this biological system cannot destroy stability. It means that there are no additional conditions from the mathematical point of view, only medical limitations should be taken into account.

\section{Construction of the Cauchy matrix}

Denote by $C_{i}(t, s)=\operatorname{col}\left\{c_{1 i}(t, s), \ldots, c_{5 i}(t, s)\right\}$ the $i$ th column of the Cauchy matrix $C(t, s)=$ $\operatorname{col}\left\{C_{1}(t, s), \ldots, C_{5}(t, s\}\right)$ (the fundamental matrix in another terminology). Consider $C_{i}(t, s)$ as a 5 -vector-function of $t$ for fixed $s$. It is known from the general theory of ordinary differential equations that $C_{i}(t, s)$ can be defined as the solution of system (3.2) satisfying the initial conditions $c_{i j}(s, s)=0$ for $i \neq j, c_{i i}(s, s)=1$.

Below we construct the Cauchy matrix of system (3.2), solving system (3.2) in the case of

$$
\begin{aligned}
& b_{2} \neq b_{1}, \quad \alpha_{1} \neq b_{1}, \quad \alpha_{1} \neq b_{2}, \\
& b_{1}^{2}+\alpha_{3} b_{3}+c_{3} \neq b_{1}\left(\alpha_{3}+b_{3}\right), \\
& b_{2}^{2}+\alpha_{3} b_{3}+c_{3} \neq b_{2}\left(\alpha_{3}+b_{3}\right), \\
& \alpha_{1}^{2}+\alpha_{3} b_{3}+c_{3} \neq \alpha_{1}\left(\alpha_{3}+b_{3}\right), \\
& \left(\alpha_{3}-b_{3}\right)^{2}>4 c_{3} .
\end{aligned}
$$

Remark 4.1 The parameters $\alpha_{3}$ and $c_{3}$ can be chosen such that inequalities (4.1) and (4.2) are fulfilled. It demonstrates that they are actually not limitations. Mathematical sense of all inequalities in (4.1), (4.2), and (4.3) will be clear below when we construct the Cauchy matrix $C(t, s)$ of system (3.2). We construct it only in the case of five different real roots of the characteristic polynomial $P(x)=0$ defined in (3.4). This will explain inequalities (4.1)-(4.3). In the case of complex roots $\lambda_{4}, \lambda_{5}$ or of the multiple roots, the construction of Cauchy matrix can be made analogously. In this paper, we do not deal with it. The case of five different real roots looks most relevant from the medical point of view. 
Solving system (3.2) in the case of (4.1)-(4.3), we obtain

$$
\left\{\begin{array}{l}
x_{1}(t)=D_{1} e^{-b_{1} t}, \\
x_{2}(t)=\frac{g_{1} D_{1} e^{-b_{1} t}}{b_{2}-b_{1}}+D_{2} e^{-b_{2} t}, \\
x_{3}(t)=E_{1} e^{\gamma_{1} t}+E_{2} e^{\gamma_{2} t}+K_{1} e^{-b_{1} t}+K_{2} e^{-b_{2} t}+K_{3} e^{-\alpha_{1} t}, \\
E_{1} e^{\gamma_{1} t}\left[\gamma_{1}+b_{3}\right]+E_{2} e^{\gamma_{2} t}\left[\gamma_{2}+b_{3}\right] \\
x_{4}(t)=-\frac{1}{c_{3}}\left(\begin{array}{c}
+K_{1} e^{-b_{1} t}\left[b_{3}-b_{1}\right]+K_{2} e^{-b_{2} t}\left[b_{3}-b_{2}\right]+K_{3} e^{-\alpha_{1} t}\left[b_{3}-\alpha_{1}\right] \\
-\frac{c_{1} g_{1} D_{1} e^{-b_{1} t}}{\left(\alpha_{1}-b_{1}\right)\left(b_{2}-b_{1}\right)}-\frac{c_{1} D_{2} e^{-b_{2} t}}{\alpha_{1}-b_{2}}-c_{1} D_{5} e^{-\alpha_{1} t}
\end{array}\right), \\
x_{5}(t)=\frac{g_{1} D_{1} e^{-b_{1} t}}{\left(\alpha_{1}-b_{1}\right)\left(b_{2}-b_{1}\right)}+\frac{D_{2} e^{-b_{2} t}}{\alpha_{1}-b_{2}}+D_{5} e^{-\alpha_{1} t}
\end{array}\right),
$$

where

$$
\begin{aligned}
& K_{1}=\frac{c_{1} g_{1} D_{1}\left[\alpha_{3}-b_{1}\right]}{\left(\alpha_{1}-b_{1}\right)\left(b_{2}-b_{1}\right)\left[b_{1}^{2}-b_{1}\left(\alpha_{3}+b_{3}\right)+\left(\alpha_{3} b_{3}+c_{3}\right)\right]}, \\
& K_{2}=\frac{c_{1} D_{2}\left[\alpha_{3}-b_{2}\right]}{\left(\alpha_{1}-b_{2}\right)\left[b_{2}^{2}-b_{2}\left(\alpha_{3}+b_{3}\right)+\left(\alpha_{3} b_{3}+c_{3}\right)\right]}, \\
& K_{3}=\frac{c_{1} D_{5}\left[\alpha_{3}-\alpha_{1}\right]}{\alpha_{1}^{2}-\alpha_{1}\left(\alpha_{3}+b_{3}\right)+\left(\alpha_{3} b_{3}+c_{3}\right)},
\end{aligned}
$$

and

$$
\gamma_{1}=\lambda_{4}, \quad \gamma_{2}=\lambda_{5}
$$

Condition (4.3) implies that $\gamma_{1}$ and $\gamma_{2}$ are different.

Denoting $X(t)=\operatorname{col}\left\{x_{1}(t), x_{2}(t), x_{3}(t), x_{4}(t), x_{5}(t)\right\}$ and $K=K_{1}+K_{2}+K_{3}$, we obtain

$$
\left\{\begin{array}{l}
x_{1}(0)=D_{1}, \\
x_{2}(0)=\frac{g_{1} D_{1}}{b_{2}-b_{1}}+D_{2}, \\
x_{3}(0)=E_{1}+E_{2}+K, \\
x_{4}(0)=-\frac{1}{c_{3}}\left(\begin{array}{c}
E_{1}\left[\gamma_{1}+b_{3}\right]+E_{2}\left[\gamma_{2}+b_{3}\right] \\
+K_{1}\left[b_{3}-b_{1}\right]+K_{2}\left[b_{3}-b_{2}\right]+K_{3}\left[b_{3}-\alpha_{1}\right] \\
-\frac{c_{1} g_{1} D_{1}}{\left(\alpha_{1}-b_{1}\right)\left(b_{2}-b_{1}\right)}-\frac{c_{1} D_{2}}{\alpha_{1}-b_{2}}-c_{1} D_{5}
\end{array}\right), \\
x_{5}(0)=\frac{g_{1} D_{1}}{\left(\alpha_{1}-b_{1}\right)\left(b_{2}-b_{1}\right)}+\frac{D_{2}}{\alpha_{1}-b_{2}}+D_{5}
\end{array}\right)
$$

(1) If $X(0)=\operatorname{col}\{1,0,0,0,0\}$, we have

$$
\left\{\begin{array}{l}
D_{1}=1, \\
D_{2}=-\frac{g_{1}}{b_{2}-b_{1}}, \\
E_{1}=-\frac{K_{1}\left[b_{3}-b_{1}\right]+K_{2}\left[b_{3}-b_{2}\right]+K_{3}\left[b_{3}-\alpha_{1}\right]-K\left[b_{3}+\gamma_{2}\right]}{\gamma_{1}-\gamma_{2}}, \\
E_{2}=\frac{K_{1}\left[b_{3}-b_{1}\right]+K_{2}\left[b_{3}-b_{2}\right]+K_{3}\left[b_{3}-\alpha_{1}\right]-K\left[\gamma_{1}+b_{3}\right]}{\gamma_{1}-\gamma_{2}}, \\
D_{5}=-\frac{g_{1}}{\left(\alpha_{1}-b_{1}\right)\left(b_{2}-b_{1}\right)}+\frac{g_{1}}{\left(\alpha_{1}-b_{2}\right)\left(b_{2}-b_{1}\right)}=\frac{g_{1}}{\left(\alpha_{1}-b_{1}\right)\left(\alpha_{1}-b_{2}\right)},
\end{array}\right.
$$


where

$$
\begin{aligned}
& K_{1}=\frac{c_{1} g_{1}\left[\alpha_{3}-b_{1}\right]}{\left(\alpha_{1}-b_{1}\right)\left(b_{2}-b_{1}\right)\left[b_{1}^{2}-b_{1}\left(\alpha_{3}+b_{3}\right)+\left(\alpha_{3} b_{3}+c_{3}\right)\right]}, \\
& K_{2}=\frac{c_{1} g_{1}\left[\alpha_{3}-b_{2}\right]}{\left(\alpha_{1}-b_{2}\right)\left(b_{2}-b_{1}\right)\left[b_{2}^{2}-b_{2}\left(\alpha_{3}+b_{3}\right)+\left(\alpha_{3} b_{3}+c_{3}\right)\right]}, \\
& K_{3}=\frac{c_{1} g_{1}\left[\alpha_{3}-\alpha_{1}\right]}{\left(\alpha_{1}-b_{1}\right)\left(\alpha_{1}-b_{2}\right)\left[\alpha_{1}^{2}-\alpha_{1}\left(\alpha_{3}+b_{3}\right)+\left(\alpha_{3} b_{3}+c_{3}\right)\right]} .
\end{aligned}
$$

The first column of the Cauchy matrix is the following:

$$
C_{1}(t, s)=\left(\begin{array}{c}
e^{-b_{1}(t-s)} \\
\frac{g_{1} e^{-b_{1}(t-s)}}{b_{2}-b_{1}}-\frac{g_{1} e^{-b_{2}(t-s)}}{b_{2}-b_{1}} \\
{\left[\begin{array}{c}
-\frac{K_{1}\left[b_{3}-b_{1}\right]+K_{2}\left[b_{3}-b_{2}\right]+K_{3}\left[b_{3}-\alpha_{1}\right]-K\left[b_{3}+\gamma_{2}\right]}{\gamma_{1}-\gamma_{2}} e^{\gamma_{1}(t-s)} \\
+\frac{K_{1}\left[b_{3}-b_{1}\right]+K_{2}\left[b_{3}-b_{2}\right]+K_{3}\left[b_{3}-\alpha_{1}\right]-K\left[\gamma_{1}+b_{3}\right]}{\gamma_{1}-\gamma_{2}} e^{\gamma_{2}(t-s)} \\
+K_{1} e^{-b_{1}(t-s)}+K_{2} e^{-b_{2}(t-s)}+K_{3} e^{-\alpha_{1}(t-s)}
\end{array}\right]} \\
-\frac{K_{1}\left[b_{3}-b_{1}\right]+K_{2}\left[b_{3}-b_{2}\right]+K K_{3}\left[b_{3}-\alpha_{1}\right]-K\left[b_{3}+\gamma_{2}\right]}{\gamma_{1}-\gamma_{2}} e^{\gamma_{1}(t-s)}\left[\gamma_{1}+b_{3}\right] \\
+\frac{K_{1}\left[b_{3}-b_{1}\right]+K_{2}\left[b_{3}-b_{2}\right]+K_{3}\left[b_{3}-\alpha_{1}\right]-K\left[\gamma_{1}+b_{3}\right]}{\gamma_{1}-\gamma_{2}} e^{\gamma_{2}(t-s)}\left[\gamma_{2}+b_{3}\right] \\
-\frac{1}{c_{3}}\left[\begin{array}{c}
\gamma_{1} \\
+K_{1} e^{-b_{1}(t-s)}\left[b_{3}-b_{1}\right]+K_{2} e^{-b_{2}(t-s)}\left[b_{3}-b_{2}\right]+K_{3} e^{-\alpha_{1}(t-s)}\left[b_{3}-\alpha_{1}\right] \\
-\frac{c_{1} g_{1} e^{-b_{1}(t-s)}}{\left(\alpha_{1}-b_{1}\right)\left(b_{2}-b_{1}\right)}+\frac{c_{1} g_{1} e^{-b_{2}(t-s)}}{\left(\alpha_{1}-b_{2}\right)\left(b_{2}-b_{1}\right)}-\frac{g_{1} c_{1} e^{-\alpha_{1}(t-s)}}{\left(\alpha_{1}-e_{1}\right)\left(\alpha_{1}-b_{2}\right)} \\
\frac{g_{1} e^{-b_{1}(t-s)}}{\left(\alpha_{1}-b_{1}\right)\left(b_{2}-b_{1}\right)}-\frac{g_{1} e^{-b_{2}(t-s)}}{\left(\alpha_{1}-b_{2}\right)\left(b_{2}-b_{1}\right)}+\frac{g_{1} e^{-\alpha_{1}(t-s)}}{\left(\alpha_{1}-b_{1}\right)\left(\alpha_{1}-b_{2}\right)}
\end{array}\right]
\end{array}\right) .
$$

(2) If $X(0)=\operatorname{col}\{0,1,0,0,0\}$, we have

$$
\left\{\begin{array}{l}
D_{1}=0, \\
D_{2}=1, \\
E_{1}=-\frac{K_{1}\left[b_{3}-b_{1}\right]+K_{2}\left[b_{3}-b_{2}\right]+K_{3}\left[b_{3}-\alpha_{1}\right]-K\left[b_{3}+\gamma_{2}\right]}{\gamma_{1}-\gamma_{2}}, \\
E_{2}=\frac{K_{1}\left[b_{3}-b_{1}\right]+K_{2}\left[b_{3}-b_{2}\right]+K_{3}\left[b_{3}-\alpha_{1}\right]-K\left[\gamma_{1}+b_{3}\right]}{\gamma_{1}-\gamma_{2}}, \\
D_{5}=-\frac{1}{\alpha_{1}-b_{2}},
\end{array}\right.
$$

where

$$
\begin{aligned}
& K_{1}=0, \\
& K_{2}=\frac{c_{1}\left[\alpha_{3}-b_{2}\right]}{\left(\alpha_{1}-b_{2}\right)\left[b_{2}^{2}-b_{2}\left(\alpha_{3}+b_{3}\right)+\left(\alpha_{3} b_{3}+c_{3}\right)\right]}, \\
& K_{3}=\frac{c_{1}\left[\alpha_{3}-\alpha_{1}\right]}{\left(b_{2}-\alpha_{1}\right)\left[\alpha_{1}^{2}-\alpha_{1}\left(\alpha_{3}+b_{3}\right)+\left(\alpha_{3} b_{3}+c_{3}\right)\right]} .
\end{aligned}
$$


The second column of the Cauchy matrix is the following:

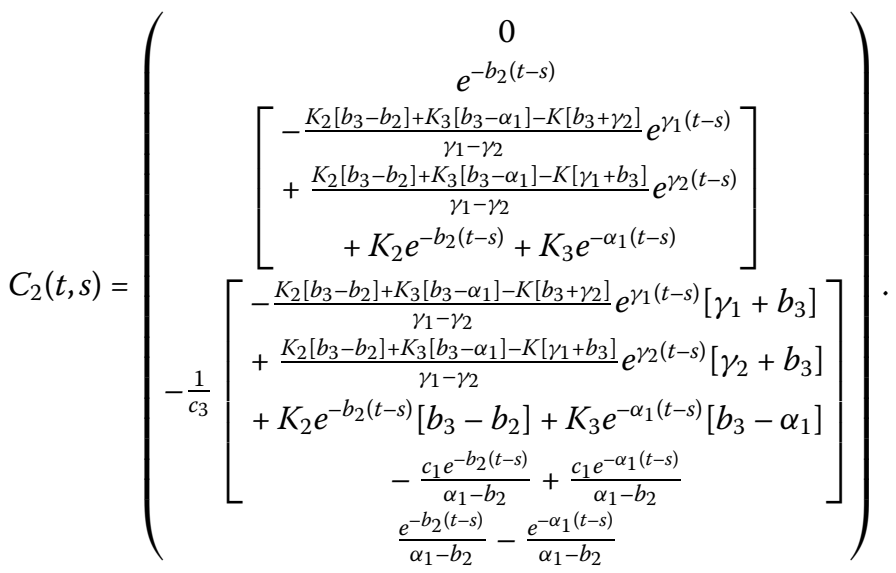

(3) If $X(0)=\operatorname{col}\{0,0,1,0,0\}$, we have

$$
\left\{\begin{array}{l}
D_{1}=0 \\
D_{2}=0 \\
E_{1}=-\frac{K_{1}\left[b_{3}-b_{1}\right]+K_{2}\left[b_{3}-b_{2}\right]+K_{3}\left[b_{3}-\alpha_{1}\right]+(1-K)\left[b_{3}+\gamma_{2}\right]}{\gamma_{1}-\gamma_{2}} \\
E_{2}=\frac{K_{1}\left[b_{3}-b_{1}\right]+K_{2}\left[b_{3}-b_{2}\right]+K_{3}\left[b_{3}-\alpha_{1}\right]+(1-K)\left[\gamma_{1}+b_{3}\right]}{\gamma_{1}-\gamma_{2}} \\
D_{5}=0
\end{array}\right.
$$

where

$$
K_{1}=0, \quad K_{2}=0, \quad K_{3}=0 .
$$

The third column of the Cauchy matrix is the following:

$$
C_{3}(t, s)=\left(\begin{array}{c}
0 \\
0 \\
-\frac{e^{\gamma_{1}(t-s)}}{\gamma_{1}-\gamma_{2}}+\frac{e^{\gamma_{2}(t-s)}}{\gamma_{1}-\gamma_{2}} \\
-\frac{1}{c_{3}}\left(-\frac{e^{\gamma_{1}(t-s)}}{\gamma_{1}-\gamma_{2}}\left[\gamma_{1}+b_{3}\right]+\frac{e^{\gamma_{2}(t-s)}}{\gamma_{1}-\gamma_{2}}\left[\gamma_{2}+b_{3}\right]\right) \\
0
\end{array}\right) .
$$

(4) If $X(0)=\operatorname{col}\{0,0,0,1,0\}$, we have

$$
\left\{\begin{array}{l}
D_{1}=0 \\
D_{2}=0 \\
E_{1}=-\frac{-c_{3}-K_{1}\left[b_{3}-b_{1}\right]-K_{2}\left[b_{3}-b_{2}\right]-K_{3}\left[b_{3}-\alpha_{1}\right]+K\left[\gamma_{2}+b_{3}\right]}{\gamma_{2}-\gamma_{1}}, \\
E_{2}=\frac{-c_{3}-K_{1}\left[b_{3}-b_{1}\right]-K_{2}\left[b_{3}-b_{2}\right]-K_{3}\left[b_{3}-\alpha_{1}\right]+K\left[\gamma_{1}+b_{3}\right]}{\gamma_{2}-\gamma_{1}} \\
D_{5}=0
\end{array}\right.
$$

where

$$
K_{1}=0, \quad K_{2}=0, \quad K_{3}=0 .
$$


The fourth column of the Cauchy matrix is the following:

$$
C_{4}(t, s)=\left(\begin{array}{c}
0 \\
0 \\
\frac{c_{3} e^{\gamma_{1}(t-s)}}{\gamma_{2}-\gamma_{1}}-\frac{c_{3} e^{\gamma_{2}(t-s)}}{\gamma_{2}-\gamma_{1}} \\
-\frac{e^{\gamma_{1}(t-s)}\left[\gamma_{1}+b_{3}\right]}{\gamma_{2}-\gamma_{1}}+\frac{e^{\gamma_{2}(t-s)}\left[\gamma_{2}+b_{3}\right]}{\gamma_{2}-\gamma_{1}} \\
0
\end{array}\right) .
$$

(5) If $X(0)=\operatorname{col}\{0,0,0,0,1\}$, we have

$$
\left\{\begin{array}{l}
D_{1}=0, \\
D_{2}=0, \\
E_{1}=-\frac{c_{1}-K_{1}\left[b_{3}-b_{1}\right]-K_{2}\left[b_{3}-b_{2}\right]-K_{3}\left[b_{3}-\alpha_{1}\right]+K\left[\gamma_{2}+b_{3}\right]}{\gamma_{2}-\gamma_{1}}, \\
E_{2}=\frac{c_{1}-K_{1}\left[b_{3}-b_{1}\right]-K_{2}\left[b_{3}-b_{2}\right]-K_{3}\left[b_{3}-\alpha_{1}\right]+K\left[\gamma_{1}+b_{3}\right]}{\gamma_{2}-\gamma_{1}}, \\
D_{5}=1,
\end{array}\right.
$$

where

$$
K_{1}=0, \quad K_{2}=0, \quad K_{3}=\frac{c_{1}\left[\alpha_{3}-\alpha_{1}\right]}{\alpha_{1}^{2}-\alpha_{1}\left(\alpha_{3}+b_{3}\right)+\left(\alpha_{3} b_{3}+c_{3}\right)} .
$$

The fifth column of the Cauchy matrix is the following:

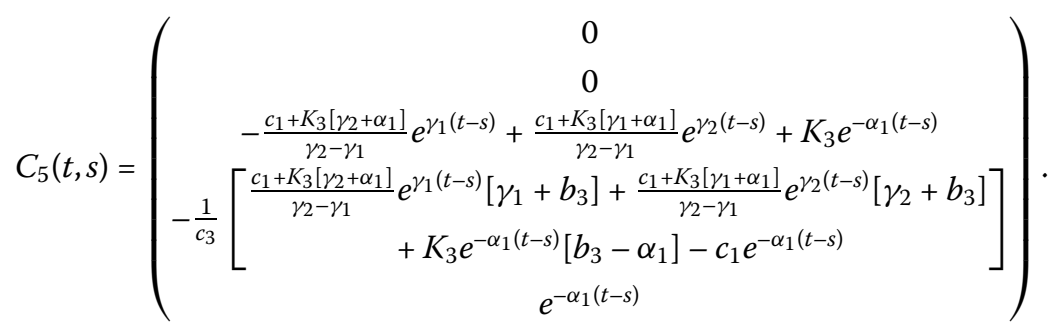

Construction of the Cauchy matrix of system with ordinary differential equations can be found, for example, in [13].

\section{Effects of changes in the right-hand side and of uncertain coefficient on the behavior of solutions}

Constructing a system, we neglect the influences of different factors that seem to be nonessential. We also cannot know exactly the values of the coefficients describing the model. The Cauchy matrix $C(t, s)$ allows us to estimate the influences of all these factors on the testosterone concentration.

Consider the systems

$$
X^{\prime}(t)-A X(t)=F(t)
$$

and

$$
Y^{\prime}(t)-A Y(t)=F(t)+\Delta F(t)
$$


where the $(5 \times 5)$ matrix $A$ is described in (3.3), $X(t)=\operatorname{col}\left(x_{1}(t), \ldots, x_{5}(t)\right)$, and $\Delta F(t)$ describes a change of the right-hand side. We assume that $F(t)$ and $\Delta F(t)$ are 5 -vectors with essentially bounded components $F_{i}(t)$ and $\Delta F_{i}(t)$. The general solution of system (5.0) has the following representation:

$$
X(t)=\int_{0}^{t} C(t, s) F(s) d s+C(t, 0) X(0)
$$

where $C(t, s)$ is the Cauchy matrix of system (5.0). In the following assertion, we estimate the difference between the solution-vector $Y(t)$ of system (5.1) and the solution $X(t)$ of system (5.0) with the same initial condition (i.e., $X(0)=Y(0)$ ).

Theorem 5.1 Under conditions (2.6), (4.1), (4.2), (4.3), system (3.2) is exponentially stable and the following inequality

$$
\|Y(t)-X(t)\| \leq\|C\|\|\Delta F(t)\|
$$

is true, where

$$
\|C\|=\max _{1 \leq j \leq 5}\left(\sup _{t \geq 0} \int_{0}^{t} \sum_{i=0}^{5}\left|C_{i j}(t, s)\right|\right) d s, \quad\|\Delta F\|=\max _{1 \leq i \leq 5} \operatorname{esssup}\left|\Delta F_{i}(t)\right| .
$$

The elements $C_{i j}(t, s)$ were obtained in paragraph 4 . The proof follows from the representation of solution of system (5.0).

Consider now the following system of equations with uncertain coefficient:

$$
\left\{\begin{array}{l}
x_{1}^{\prime}(t)+b_{1} x_{1}(t)=0 \\
x_{2}^{\prime}(t)+b_{2} x_{2}(t)-g_{1} x_{1}(t)=0 \\
x_{3}^{\prime}(t)+b_{3} x_{3}(t)-c_{1} \int_{0}^{t} e^{-\alpha_{1}(t-s)} x_{2}(s) d s+\left(c_{3}+\Delta c_{3}(t)\right) \int_{0}^{t} e^{-\alpha_{3}(t-s)} x_{3}(s) d s \\
\quad=f(t)
\end{array}\right.
$$

A "noise" in this parameter $\Delta c_{3}(t)$ can be a result of individual conditions of the patient. For example, assimilation of a drug in the body of different patients can have different speeds. System (5.2) of integro-differential equations can be reduced to the following system of ordinary differential equations:

$$
\left\{\begin{array}{l}
x_{1}^{\prime}(t)+b_{1} x_{1}(t)=0 \\
x_{2}^{\prime}(t)+b_{2} x_{2}(t)-g_{1} x_{1}(t)=0 \\
x_{3}^{\prime}(t)+b_{3} x_{3}(t)-c_{1} x_{5}(t)+\left(c_{3}+\Delta c_{3}(t)\right) x_{4}(t)=f(t) \\
x_{4}^{\prime}(t)+\alpha_{3} x_{4}(t)-x_{3}(t)=0 \\
x_{5}^{\prime}(t)+\alpha_{1} x_{5}(t)-x_{2}(t)=0
\end{array}\right.
$$

where $x_{4}(0)=0, x_{5}(0)=0$.

We can write our system in the following form:

$$
X^{\prime}=A X+(\Delta A) X+F(t)
$$


where

$$
X(t)=\left(\begin{array}{c}
x_{1}(t) \\
x_{2}(t) \\
x_{3}(t) \\
x_{4}(t) \\
x_{5}(t)
\end{array}\right), \quad \Delta A(t)=\left(\begin{array}{ccccc}
0 & 0 & 0 & 0 & 0 \\
0 & 0 & 0 & 0 & 0 \\
0 & 0 & 0 & -\Delta c_{3}(t) & 0 \\
0 & 0 & 0 & 0 & 0 \\
0 & 0 & 0 & 0 & 0
\end{array}\right), \quad F(t)=\left(\begin{array}{c}
0 \\
0 \\
f(t) \\
0 \\
0
\end{array}\right) .
$$

The general solution of the auxiliary system

$$
X^{\prime}-A X=Z
$$

can be represented in the following form:

$$
X(t)=\int_{0}^{t} C(t, s) Z(s) d s+C(t, 0) X(0)
$$

Finally, combining (5.5) and (5.4), we have

$$
Z=(\Delta A) X+F(t)
$$

whence, using expression (5.6), we arrive at

$$
Z(t)-(\Delta A)(t) \int_{0}^{t} C(t, s) Z(s) d s=F(t)+(\Delta A(t)) C(t, 0) X(0),
$$

which can be written in the operator form as follows:

$$
Z(t)=(\Omega Z)(t)+\tilde{F}(t)
$$

where $\tilde{F}$ is the right-hand side in equation (5.7). It is clear from Theorem 3.1 that $\tilde{F} \in L_{\infty}^{5}$, $L_{\infty}^{5}$ is the space of measurable essentially bounded five-component vector-functions, and

$$
\Omega: L_{\infty}^{5} \rightarrow L_{\infty}^{5}, \quad(\Omega Z)(t)=(\Delta A)(t) \int_{0}^{t} C(t, s) Z(s) d s
$$

Below we estimate the norm $\|\Omega\|$ of the operator $\Omega$. It is clear that

$$
\|\Omega\| \leq \max _{1 \leq j \leq 5}\left(\sup _{t \geq 0} \int_{0}^{t} \sum_{i=0}^{5}\left|(\triangle A(t) C(t, s))_{i j}\right|\right) d s .
$$


Let us denote $\left.Q_{j}=\operatorname{esssup}_{t \geq 0} \int_{0}^{t} \sum_{i=0}^{5}\left|(\triangle A(t) C(t, s))_{i j}\right|\right) d s, \triangle c_{3}^{*}=\operatorname{esssup}_{t \geq 0}\left|\triangle c_{3}(t)\right|$, and

$$
\begin{aligned}
& Q_{1}^{*}=\frac{\Delta c_{3}^{*}}{\left|c_{3}\right|}\left(\begin{array}{c}
\left|\frac{K_{1}\left[b_{3}+b_{1}\right]+K_{2}\left[b_{3}+b_{2}\right]+K_{3}\left[b_{3}+\alpha_{1}\right]+K\left[b_{3}+\gamma_{2}\right]}{\gamma_{1}-\gamma_{2}} \frac{\gamma_{1}+b_{3}}{\gamma_{1}}\right| \\
+\left|\frac{K_{1}\left[b_{3}+b_{1}\right]+K_{2}\left[b_{3}+b_{2}\right]+K_{3}\left[b_{3}+\alpha_{1}\right]+K\left[\gamma_{1}+b_{3}\right]}{\gamma_{1}-\gamma_{2}} \frac{\gamma_{2}+b_{3}}{\gamma_{2}}\right| \\
+\left|\frac{K_{1}\left[b_{3}+b_{1}\right]}{b_{1}}\right|+\left|\frac{K_{2}\left[b_{3}+b_{2}\right]}{b_{2}}\right|+\left|\frac{K_{3}\left[b_{3}+\alpha_{1}\right]}{\alpha_{1}}\right| \\
+\left|\frac{c_{1} g_{1}}{b_{1}\left(\alpha_{1}-b_{1}\right)\left(b_{2}-b_{1}\right)}\right|+\left|\frac{c_{1} g_{1}}{b_{2}\left(\alpha_{1}-b_{2}\right)\left(b_{2}-b_{1}\right)}\right|+\left|\frac{g_{1} c_{1}}{\alpha\left(\alpha_{1}-b_{1}\right)\left(\alpha_{1}-b_{2}\right)}\right|
\end{array}\right), \\
& \begin{array}{c}
Q_{2}^{*}=\frac{\Delta c_{3}^{*}}{\left|c_{3}\right|}\left(\begin{array}{c}
\left|\frac{K_{2}\left[b_{3}+b_{2}\right]+K_{3}\left[b_{3}+\alpha_{1}\right]+K\left[b_{3}+\gamma_{2}\right]}{\gamma_{1}-\gamma_{2}} \frac{\gamma_{1}+b_{3}}{\gamma_{1}}\right| \\
+\left|\frac{K_{2}\left[b_{3}+b_{2}\right]+K_{3}\left[b_{3}+\alpha_{1}\right]+K\left[\gamma_{1}+b_{3}\right]}{\gamma_{1}-\gamma_{2}} \frac{\gamma_{2}+b_{3}}{\gamma_{2}}\right| \\
+\left|\frac{K_{2}\left[b_{3}+b_{2}\right]}{b_{2}}\right|+\left|\frac{b_{3}+\alpha_{1}}{\alpha_{1}}\right| \\
+\left|\frac{c_{1}}{b_{2}\left(\alpha_{1}-b_{2}\right)}\right|+\left|\frac{c_{1}}{\alpha_{1}\left(\alpha_{1}-b_{2}\right)}\right|
\end{array}\right), \\
Q_{3}^{*}=\frac{\Delta c_{3}^{*}}{\left|c_{3}\right|}\left(\left|\frac{\gamma_{1}+b_{3}}{\gamma\left(\gamma_{1}-\gamma_{2}\right)}\right|+\left|\frac{\gamma_{2}+b_{3}}{\gamma_{2}\left(\gamma_{1}-\gamma_{2}\right)}\right|\right), \\
Q_{5}^{*}=\Delta c_{3}^{*}\left(\left|\frac{\gamma_{1}+b_{3}}{\gamma_{1}\left(\gamma_{2}-\gamma_{1}\right)}\right|+\left|\frac{\gamma_{2}+b_{3}}{\gamma_{2}\left(\gamma_{2}-\gamma_{1}\right)}\right|\right), \\
+\frac{c_{3}^{*}}{\left|c_{3}\right|}\left(\begin{array}{c}
\left|\frac{c_{1}+K_{3}\left[\gamma_{2}+\alpha_{1}\right]}{\gamma_{2}-\gamma_{1}} \frac{\gamma_{1}+b_{3}}{\gamma_{1}}\right|+\left|\frac{c_{1}+K_{3}\left[\gamma_{1}+\alpha_{1}\right]}{\gamma_{2}-\gamma_{1}} \frac{\gamma_{2}+b_{3}}{\gamma_{2}}\right| \\
+\left|\frac{K_{3}\left[b_{3}-\alpha_{1}\right]}{\alpha_{1}}\right|+\left|\frac{c_{1}}{\alpha_{1}}\right|
\end{array}\right) .
\end{array}
\end{aligned}
$$

It is clear from the estimates of the elements of the Cauchy matrix that $Q_{j} \leq Q_{j}^{*}$ for $1 \leq j \leq 5$.

Theorem 5.2 Let conditions (2.6), (4.1), (4.2), (4.3) and the inequality $\max _{1 \leq j \leq 5}\left\{Q_{j}^{*}\right\}<1$ be fulfilled, then system (5.3) with uncertain coefficient is also exponentially stable.

Acknowledgements

This paper is a part of the PhD thesis by Marina Bershadsky and a part of the Master thesis by Olga Pinhasov. They thank Ariel University for a possibility to perform the study there.

\section{Funding}

There is no funding.

\section{Availability of data and materials}

All results are available for everyone.

\section{Competing interests}

There are no competing interests.

\section{Authors' contributions}

All authors worked together on the paper. All authors read and approved the final manuscript.

\section{Authors' information}

Professor Alexander Domoshnitsky and Dr. Irina Volinsky are the staff members of this Department, Olga Pinhasov is a student of Master Degree, Marina Bershadsky is a PhD student.

\section{Publisher's Note}

Springer Nature remains neutral with regard to jurisdictional claims in published maps and institutional affiliations.

Received: 5 June 2019 Accepted: 15 November 2019 Published online: 04 December 2019

\section{References}

1. Agarwal, R.P., Berezansky, L., Braverman, E., Domoshnitsky, A.: Nonoscillation Theory of Functional Differential Equations with Applications. Springer, New York (2012)

2. Bekiaris-Liberis, N., Krstic, M.: Lyapunov stability of linear predictor feedback for distributed input delays. IEEE Trans. Autom. Control 56(3), 655-660 (2011)

3. Cartwright, M., Husain, M.: A model for the control of testosterone secretion. J. Theor. Biol. 123, $239-250$ (1986) 
4. Churilov, A., Medvedev, A.: Discrete-time map for an impulsive Goodwin oscillator with a distributed delay. Math. Control Signals Syst. 28(1), 1-22 (2016)

5. Churilov, A., Medvedev, A., Mattson, P.: Periodical solutions in a pulse-modulated model of endocrine regulation with time-delay. IEEE Trans. Autom. Control 59(3), 728-733 (2014)

6. Churilov, A., Medvedev, A., Mattson, P.: Discrete-time modelling of a hereditary impulsive feedback system. In: Proceeding of the 53rd IEEE Conference of Decision Control, Los Angeles, December 15-17, pp. 765-770 (2014)

7. Das, P., Roy, A.B., Das, A.: Stability and oscillation of a negative feedback delay model for the control of testosterone secretion. Biosystems 32(1), 61-69 (1994)

8. Domoshnitsky, A.: Exponential stability of convolution integro-differential equations. Funct. Differ. Equ. 5, 445-455 (1998)

9. Domoshnitsky, A., Fridman, E.: A positivity-based approach to delay-dependent stability of systems with large time-varying delays. Syst. Control Lett. 97, 139-148 (2016)

10. Domoshnitsky, A., Shklyar, R.: Positivity for non-Metzler systems and its applications to stability of time-varying delay system. Syst. Control Lett. 118, 44-51 (2018)

11. Domoshnitsky, A., Volinsky, I., Bershadsky, M.: Around the model of infection disease: the Cauchy matrix and its properties. Symmetry 11(8), 1016 (2019). https://doi.org/10.3390/sym1 1081016. New developments in Functional and Fractional Differential Equations and in Lie Symmetry

12. Domoshnitsky, A., Volinsky, I., Polonsky, A.: Stabilization of third order differential equation by delay distributed feedback control. Math. Slovaca 69(5), 341 (2019)

13. Domoshnitsky, A., Volinsky, I., Polonsky, A., Sitkin, A.: Practical constructing the Cauchy function of integro-differential equation. Funct. Differ. Equ. 23(3-4), 109-117 (2016)

14. Domoshnitsky, A., Volinsky, I., Polonsky, A., Sitkin, A.: Stabilization by delay distributed feedback control. Math. Model. Nat. Phenom. 12(6), 91-105 (2017)

15. Farina, L., Rinaldi, S.: Positive Linear System: Theory and Applications. Wiley, New York (2000)

16. Ferry, J.D.: Viscoelastic Properties of Polymers. Wiley, New York (1970)

17. Goebel, G., Munz, U., Allgower, F: Stabilization of linear systems with distributed input delay. In: 2010 American Control Conference, pp. 5800-5805. (2010)

18. Gu, K., Kharitonov, V.L., Chen, J.: Stability of Time Delay Systems. Birkhauser Boston, Cambridge (2003)

19. Gyori, I., Hartung, F.: Fundamental solution and asymptotic stability of linear delay differential equations. Dyn. Contin. Discrete Impuls. Syst. 13(2), 261-287 (2006)

20. Haddad, W.M., Chellaboina, V., Hui, Q.: Nonnegative and Compartmental Dynamical Systems. Princeton University Press, Princeton (2010)

21. Keenn, D.M., Vedhulis, J.D.: A biomathematical model of time-delayed feedback in the human male hypothalamic-pituitary-Leyding cell axis. Am. J. Physiol: Endocrinol. Metab. 275(1), 157-176 (1998)

22. Krasnosel'skii, M.A., Vainikko, G.M., Zabreiko, P.P., Rutitskii, J.B., Stezenko, V.J.: Approximate Methods for Solving Operator Equations. Nauka, Moscow (1969)

23. Mazenc, F., Niculescu, S.I., Bekaik, M.: Stabilization of time-varying nonlinear systems with distributed input delay by feedback of plant's state. IEEE Trans. Autom. Control 58(1) (2013)

24. Mukhopadhyya, B., Bhattacharyya, R.: A delayed mathematical model for testosterone secretion with feedback control mechanism. Int. J. Math. Math. Sci. 2004, 105-115 (2004)

25. Murray, J.D.: Mathematical Biology: I. An Introduction, 3rd edn. Springer, Berlin (2001)

26. Ngoc, P.H.A.: Stability of positive differential systems with delay. IEEE Trans. Autom. Control 58(1), 203-209 (2013)

27. Pinhasov, O.: Positivity - based stability analysis in testosterone regulation using distributed feedback control. Russ. J. Biomech. (2019) (accepted)

28. Smith, H.L:: Monotone Dynamical Systems: An Introduction to the Theory of Competitive and Cooperative Systems. Am. Math. Soc., Providence (1995)

29. Smith, W.R.: Qualitative mathematical models of endocrine systems. Am. J. Physiol. 245(4), 473-477 (1989)

\section{Submit your manuscript to a SpringerOpen ${ }^{\circ}$ journal and benefit from:}

- Convenient online submission

- Rigorous peer review

- Open access: articles freely available online

- High visibility within the field

- Retaining the copyright to your article

Submit your next manuscript at $\gg$ springeropen.com 